\title{
SiC THz Sensors for Harsh Environment Applications
}

\author{
Ibrahim Abdel-Motaleb*, Sai Dittakavi \\ Department of Electrical Engineering, Northern Illinois University, DeKalb, USA \\ Email: ^ibrahim@niu.edu
}

How to cite this paper: Abdel-Motaleb, I. and Dittakavi, S. (2021) SiC THz Sensors for Harsh Environment Applications. Open Journal of Antennas and Propagation, 9, 45-55. https://doi.org/10.4236/ojapr.2021.93004

Received: August 13, 2021

Accepted: September 27, 2021

Published: September 30, 2021

Copyright $\odot 2021$ by author(s) and Scientific Research Publishing Inc. This work is licensed under the Creative Commons Attribution International License (CC BY 4.0).

http://creativecommons.org/licenses/by/4.0/

\begin{abstract}
Gold $(\mathrm{Au})$ and ITO bow-tie antenna sensors built on silicon carbide (SiC) substrates are designed and analyzed using the Comsol Multiphysics program. The study shows that the output electric field across the antenna gap decreases with gap separation but increases with metal thickness. The antenna width does not directly affect the output, but it indirectly increases the output as a result of increased radiation absorption and conductivity. The length does not affect the strength of the output, but it affects the resonant frequency. The output field from ITO antennas is found to be much lower than that from $\mathrm{Au}$ antennas, which can be attributed, in part, to the lower conductivity of ITO. Using the bow-tie antenna structure, it is possible to build either narrowband or wideband antenna or sensor arrays, for $\mathrm{THz}$ applications.
\end{abstract}

\section{Keywords}

Silicon Carbide, SiC, Sensor, Terahertz, THz, ITO, Numerical Analysis, COMSOL

\section{Introduction}

Harsh environments, such space and battlefields, may suffer from high radiation, high temperatures, and abrasive atmospheres. This requires devices that can withstand such abusive environments. THz sensors and antennas are among the widely used devices in space exploration and battlefields. For example, they are used to observe terrestrial object movements and their chemical compositions through photon emission and red-shift observation. On the battlefield, they can be used for communications and imaging. The systems used in such applications are complex, which may require the integration with optical and electronic devices and systems.

The terahertz spectrum from $0.3 \mathrm{THz}-3 \mathrm{THz}$ is known as the sub-millimeter 
range with a wavelength from $0.1 \mathrm{~mm}$ to $1 \mathrm{~mm}$ [1]. This wavelength region is located between the micro-wave regions and infra-red regions. Hence, $\mathrm{THz}$ signals can be treated as a microwave signal or as an infra-red signal. THz devices can be used in many applications such as sensing, medical diagnostics, imaging, communications, security, and scientific instrumentations [2] [3]. It can, also, be used in many industrial applications, such as plastic inspection, paint and coating layer evaluation, industrial quality control, material research, gas sensing, hydration monitoring, and ultrafast dynamics [4]. Because $\mathrm{THz}$ can be treated as electromagnetic microwave and infrared signals, $\mathrm{THz}$ devices can be considered both sensors and antennas. Hence the words "sensor" and "antenna" are used here interchangeably.

One of the main disadvantages of using X-ray is that it is an ionizing radiation, which can pose a great harm to the tissues and DNA sequences. On the other hand, $\mathrm{THz}$ radiation has the non-ionizing property, which makes it very safe for use in medical applications.

For high-temperature environments and space applications, wide-bandgap semiconductor substrates should be used to build the $\mathrm{THz}$ sensor or antenna. SiC can satisfy these conditions for several reasons: first, it is a wideband gap material $(\mathrm{Eg}=2.3$ to $3.2 \mathrm{eV})$. This makes $\mathrm{SiC}$ an excellent insulator; second, it can operate at very high temperatures; third, it has a high thermal conductivity; fourth, it can be used to integrate electronics and optoelectronic systems. Therefore, $\mathrm{SiC}$ is one of the best candidates for space and defense applications.

$\mathrm{THz}$ antennas and sensors can be built using many configurations and geometries. For example, they can be built using metamaterial absorbers [5] [6] or using a spiral structure. In this study, the bow-tie shape is used for its simplicity and efficiency. In this design, metals in the shape of bow-tie are deposited on top of undoped insulating $\mathrm{SiC}$ substrates. The performance of the antenna is affected by many factors, such as the dielectric strength of the substrate, the conductivity of the metal, and the geometry of the device. Silica is used to build $\mathrm{THz}$ antennas for its compatibility with Si technology. Such antennas have been studied by others, and their performances have been reported in the literatures [2] [3].

The metal used to build $\mathrm{THz}$ antennas can be gold ( $\mathrm{Au}$ ), copper, or any high conductivity materials. However, $\mathrm{Au}$ is normally chosen because of its low resistivity and chemical inertness. But, Au metal is opaque, which limits the ability for vertical integration with optoelectronic systems. It may be advantageous, in some applications, to use transparent conductors to allow for integration with optical systems, if needed. Indium-Tin-Oxide (ITO) can provide the required high conductivity and transparency due to its wide energy gap, which provides transparency and radiation hardness. The high conductivity of ITO is a result of its degeneracy, where the Fermi energy lays in the conduction band [7].

To the best of our knowledge, a THz bow-tie antenna using SiC, suitable for a hard environment with the ability for vertical integration with optical devices, has not been reported. This work is a step towards this goal. Therefore, this re- 
port focuses on designing, characterizing, and optimizing two sets of antenna sensors using Au and ITO on SiC substrates in the $0.3-5 \mathrm{THz}$ range. The $4 \mathrm{H}-\mathrm{SiC}$ material is chosen for its high quality, wide energy gap, transparency, and high thermal conductivity coefficient [8].

\section{Methodology}

COMSOL Multiphysics program is used to design and analyze the antennas under study. The bow-tie shape is attractive because of its simple structure and its ability to provide enhanced performance. Bow-ties antenna studies have been reported by many research groups for different applications [2] [3] [9] [10]. The geometry of the device is basically the shape of a bow-tie, where it is composed of two metal triangles with a nano-gap between their vertices. Figure 1 shows the bow-tie antenna used in our study. The base design is an antenna with a length of $16 \mu \mathrm{m}$, width of $9 \mu \mathrm{m}$, thickness of $1 \mu \mathrm{m}$, and gap between the two triangles of $100 \mathrm{~nm}$.

The geometry of the antenna, shown in Figure 1(b), is created in COMSOL. The figure shows the strength of the electric field, which is the highest across the gap. Figure 2 shows a 3-D view of the geometry used in Comsol to simulate the performance of the antennas. As can be seen in the figure, the antenna is placed on top of $20 \mu \mathrm{m} \mathrm{SiC} \mathrm{substrate.} \mathrm{Initially,} \mathrm{an} \mathrm{air} \mathrm{layer} \mathrm{of} 20 \mu \mathrm{m}$ was placed above the metal and under the substrate. However, during the first round of device simulation, it was discovered that the signal is reflected at the surface of the air layer, causing unwanted secondary excitations. To avoid such reflections at the boundaries, a Perfectly Matched Layer (PML) with thickness of $10 \mu \mathrm{m}$ is placed on the top of the air layer and under the substrate, as shown in the figure [10]. PML is not a boundary condition, just an absorber. A $1 \mathrm{~V} / \mathrm{m}$ Electromagnetic wave is applied vertically on the device using a COMSOL feature port in the z-direction. This port is excited with an electric source placed at $20 \mu \mathrm{m}$ above the

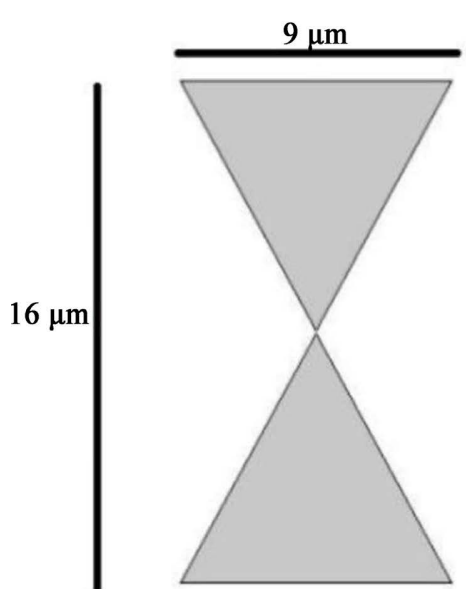

(a)

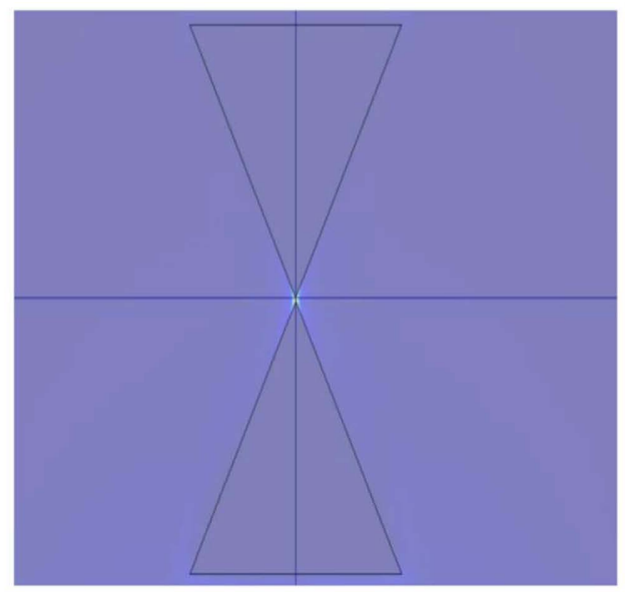

(b)

Figure 1. (a) Geometry of the bow-tie nano-antenna sensor used in the study; (b) COMSOL simulation of the electric filed intensity showing the highest value is across the gap. 


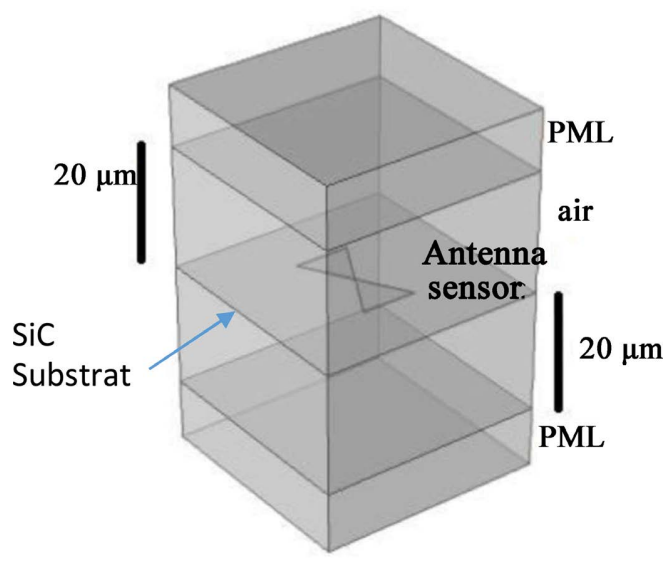

Figure 2. A 3D view of simulation domain in COMSOL with a Perfectly Matched Layer (PML) on the top and at the bottom.

sensor metal, but just below the upper PML layer.

The simulation is performed using frequency dependent parameters. The dependence of the dielectric constant on the frequency is obtained from Drude model [6].

$$
\varepsilon(\omega)=\varepsilon_{\infty}-\frac{\omega_{p}^{2}}{\omega^{2}-j \omega \cdot \omega_{\tau}}
$$

where $\omega$ is the radial frequency, $\varepsilon_{\infty}$ is the contribution of the bound electrons to the relative dielectric constant, $\omega_{p}$ is the plasma frequency, and $\omega_{\tau}$ is the damping frequency. The plasma frequency is obtained from:

$$
\omega_{p}=\sqrt{q^{2} n / m^{*} \varepsilon_{0}}
$$

where $n$ is the free electron density in the conduction band, $q$ is the electron charge, $\mathcal{E}_{0}$ is the permittivity of the free space, and $m^{*}$ is the electron effective mass. COMSOL uses Equation (1) to obtain the complex dielectric constant as a function of the frequency [11]. In the $\mathrm{THz}$ range, the imaginary part may be significant, especially in case of conducting materials.

The electric field detected across the gap is the output of the antenna. To obtain the electric field in COMSOL, Maxwell equation is solved. This equation is:

$$
\nabla * \mu_{r}^{-1}(\nabla * E)-k_{0}^{2} \varepsilon_{r} E=0
$$

where $\mu_{r}$ is the relative permeability, and $\mathcal{E}_{r}$ is the relative dielectric constant, and $k_{0}$ is the wave number $=(\omega / c)$, and $c$ is the speed of light.

The electric field is typically concentrated at the tip of the gap due to Coulomb field. In this case, the gap acts as a capacitor [12]. Therefore, the gap size is a critical parameter; therefore, its effect on the output field is investigated.

The application of periodic electromagnetic waves on a symmetrical structure, such as antennas, should yield outputs with some periodicity. This has been explained by [13], where a maximum peak is created at a fundamental resonant frequency, $f_{0}$, with other secondary peaks at other frequencies. On the other hand, the secondary frequencies take-place at the fractions and the multiples of 
the fundamental frequency, i.e. at $f_{0} / n$ and at $n f_{0}$, where $n$ is an integer. This means if the fundamental frequency is $f_{0}$ for an antenna, it is expected to have peaks at $f_{0} / n, \ldots, f_{0} / 3, f_{0} / 2, f_{0}, 2 f_{0}, 3 f_{0}, \ldots, n f_{0}$.

The fundamental frequency is obtained from the relation:

$$
f_{0}=\frac{c}{2 * L * \sqrt{\varepsilon_{\text {eff }}}}
$$

where, $c$ is the speed of light, $L$ is the length of the antenna, and $\varepsilon_{\text {eff }}$ is the effective dielectric constant at $f_{0}$.

Considering the Au/Silica antenna shown in Figure 3, Equation (4) gives $f_{0}=$ 6.629 THz. This value lies outside the frequency range used in this study. However, according to [13], there is a secondary resonance frequency at $f_{0} / 2$ or 3.35 THz. This is the exact value obtained from [3] and shown in Figure 3.

\section{Results and Discussion}

To ensure the accuracy of our simulation method, we simulated the Au/silica bow-tie antenna reported in [3]; see Figure 3. Our simulation for the same antenna is shown in Figure 4. In this study, the conductivity of Au is assumed to be $45.6 \times 10^{6} \mathrm{~S} / \mathrm{m}$ and its dielectric constant to be $-8.49+1.62 \mathrm{i}$. For silica, the

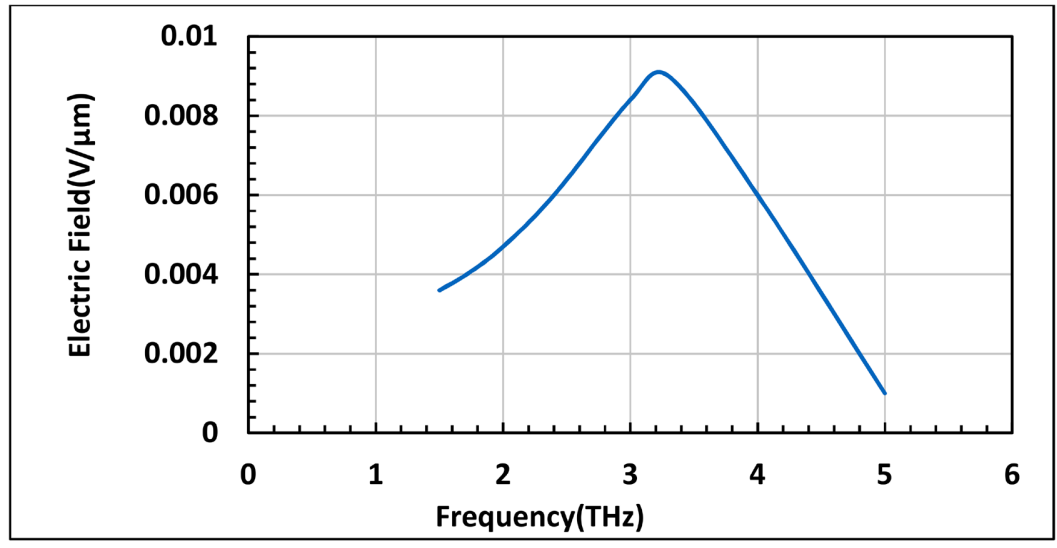

Figure 3. Output of Au/Silica antenna reported in [3].

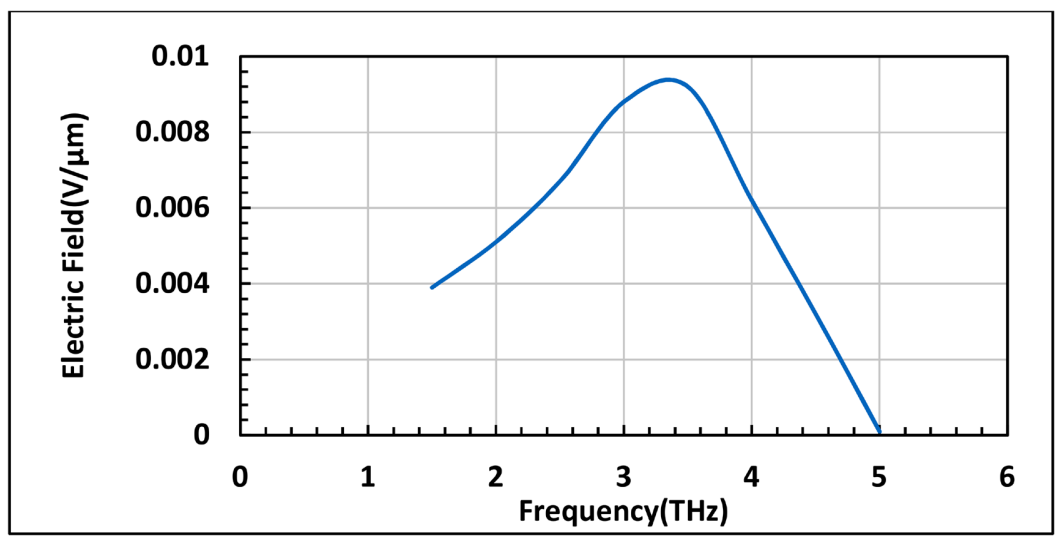

Figure 4. Output of Au/Silica antenna from this study. 
dielectric constant is assumed to be 2.03. As can be seen from Figure 3 and Figure 4, our results coincide with the results reported in [3]. Both results show resonant frequencies of about $3.35 \mathrm{THz}$, and this is a proof that our simulation provides credible results.

After ensuring the credibility of our simulation results, two types of antennas are investigated: $\mathrm{Au} / \mathrm{SiC}$ and ITO/SiC. The high frequency dielectric constant for $\mathrm{SiC}$ was taken to be $\varepsilon_{\infty}=10$. The conductivity and complex dielectric for $\mathrm{Au}$ is mentioned above. The conductivity for the ITO is taken to be $1.3 \times 10^{4} \mathrm{~S} / \mathrm{m}$ and the dielectric constant to be $3.37+0.01$. The performances for these two antennas are simulated with different dimensions.

The first critical dimension to be investigated is the gap separation. As can be seen from Figure 5 and Figure 6, the output electric field increases with the decrease of the gap separation. This is expected, since the gap acts as a capacitor, where the electric field increases with the decrease of the dielectric thickness, or the gap thickness. For $\mathrm{Au} / \mathrm{SiC}$ antenna, Figure 5, the electric field increased

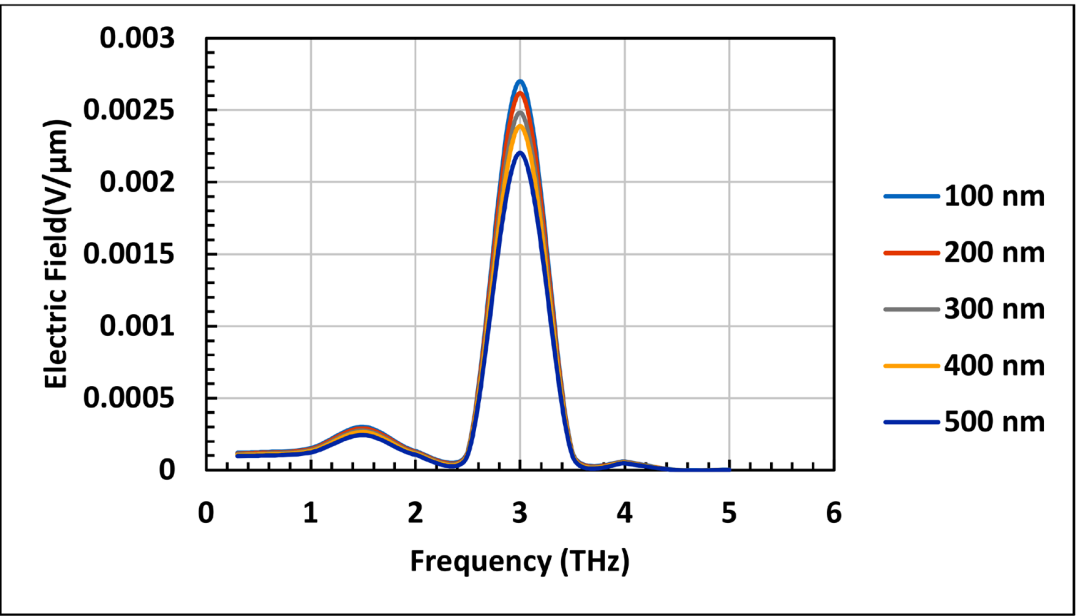

Figure 5. Electric field resulted from $\mathrm{Au} / \mathrm{SiC}$ antenna at various gap lengths between elements.

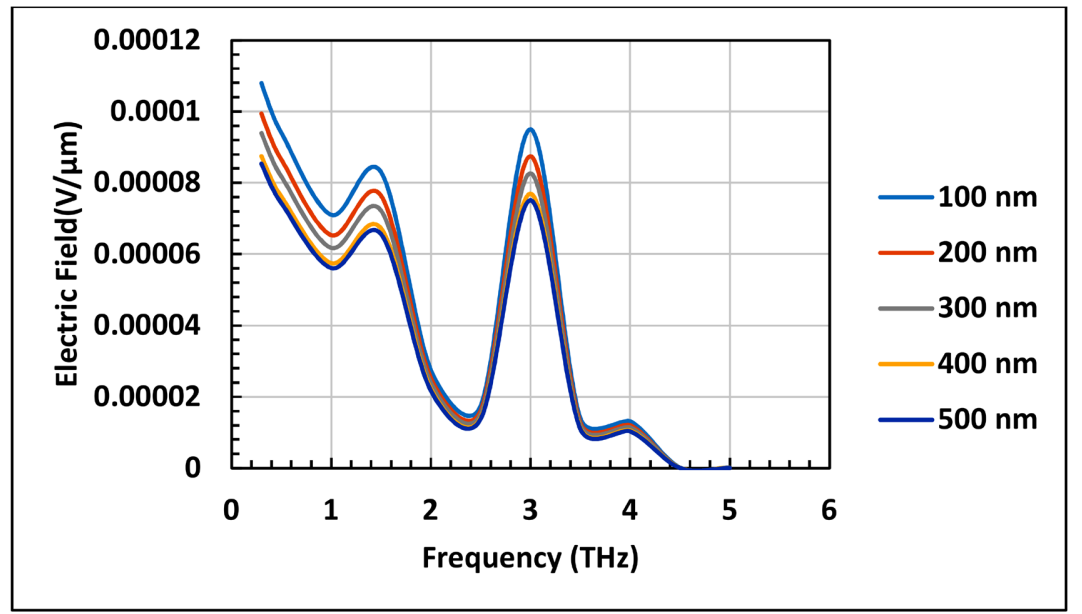

Figure 6. Electric field resulted from ITO/SiC antenna at various gap separations. 
from $2.2 \mathrm{mV} / \mu \mathrm{m}$ for a $500 \mathrm{~nm}$ gap to $2.7 \mathrm{mV} / \mu \mathrm{m}$ for a $100 \mathrm{~nm}$ gap at the peak frequency of $3 \mathrm{THz}$. For ITO/SiC antenna, although the behavior is the same, the electric field is greatly reduced. In this case, the electric field increased from 7.5 $\times 10^{-2} \mathrm{mV} / \mu \mathrm{m}$ to $9.5 \times 10^{-2} \mathrm{mV} / \mu \mathrm{m}$ for the same gap separation values. This value is about $3.5 \%$ of the values for $\mathrm{Au} / \mathrm{SiC}$ antennas. The low magnitude of the output for ITO antennas compared with Au antennas can be attributed, in part, to the lower conductivity of ITO which is $1.3 \times 10^{4} \mathrm{~S} / \mathrm{m}$ compared with $45.6 \times$ $10^{6} \mathrm{~S} / \mathrm{m}$, for $\mathrm{Au}$.

The output field is simulated next for thicknesses of $0.2 \mu \mathrm{m}, 0.4 \mu \mathrm{m}, 1 \mu \mathrm{m}, 2$ $\mu \mathrm{m}$, and $5 \mu \mathrm{m}$, while all other parameters remain as those of the base design mentioned in the Analysis section. Figure 7 and Figure 8 show that the output electric field increases as the thickness increases. This is expected, since higher thickness results in higher conductivity for the metal, and this reduces the output loss. Figure 7 shows that the output field for $\mathrm{Au} / \mathrm{SiC}$ antennas at the $3 \mathrm{THz}$ peak increased from $2.19 \mathrm{mV} / \mu \mathrm{m}$ for a thickness of $0.2 \mu \mathrm{m}$ to $3.24 \mathrm{mV} / \mu \mathrm{m}$ for a thickness of $5 \mu \mathrm{m}$. This is compared with a change of $8 \times 10^{-2} \mathrm{mV} / \mu \mathrm{m}$ to 0.106

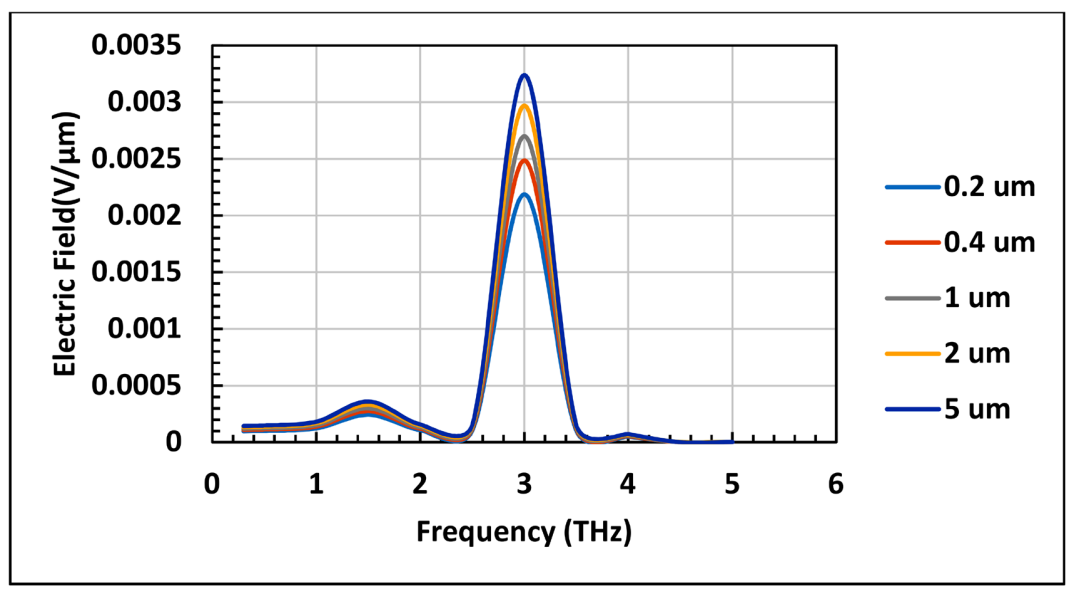

Figure 7. Electric field resulted from $\mathrm{Au} / \mathrm{SiC}$ antenna for different thicknesses.

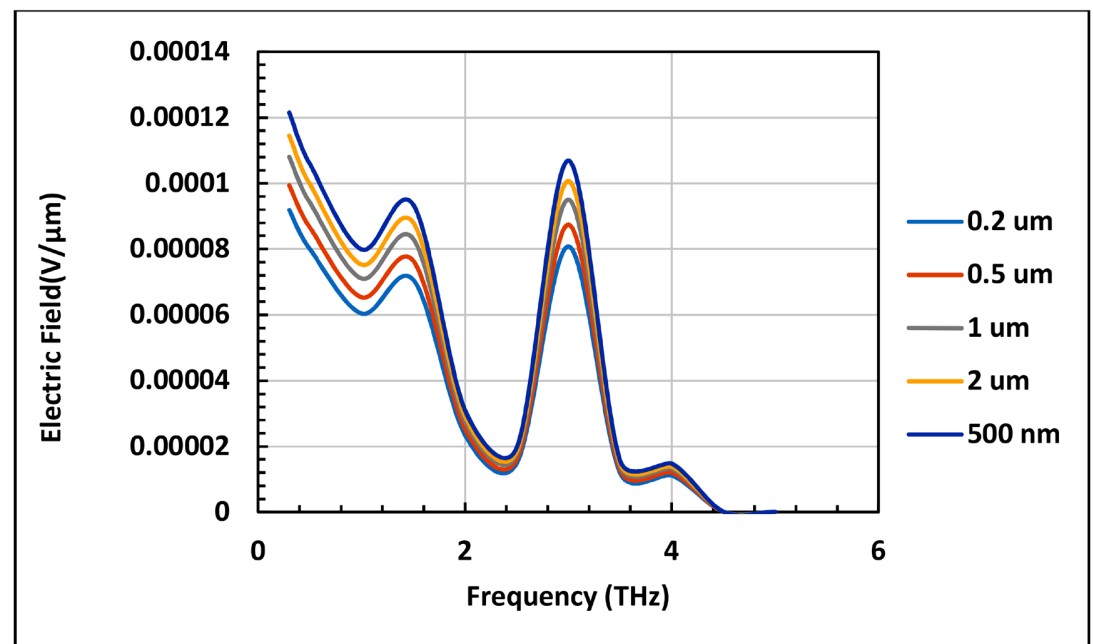

Figure 8. Electric field resulted from ITO/SiC antenna for different thicknesses. 
$\mathrm{mV} / \mu \mathrm{m}$ to for the same thicknesses. Again the low output field can also be attributed to the low conductivity of ITO compared with Au. For the ITO antenna the secondary peak at $1.5 \mathrm{THz}$ has a magnitude close to the peak at $3 \mathrm{THz}$. This may be due to the nature of ITO which is a semiconductor not a pure metal such as $\mathrm{Au}$. However, this issue needs more investigation to uncover the cause of this behavior, and its relation to the conductor and substrate properties and the interface between them.

When the width is changed to $2 \mu \mathrm{m}, 4 \mu \mathrm{m}, 9 \mu \mathrm{m}, 10 \mu \mathrm{m}$, and $12 \mu \mathrm{m}$, it was expected that the output field will remain constant. The reason for this expectation is the fact that the electric field is applied along the length and perpendicular to the width. This means the width has no effect on the output. However, the analysis shows that the electric field increases with the increase of the width, Figure 9 and Figure 10. We believe that this increase can be attributed to two indirect effects of the width increase. The first is the increase of the area of the antenna, which leads to the increase of radiation absorption and consequently to

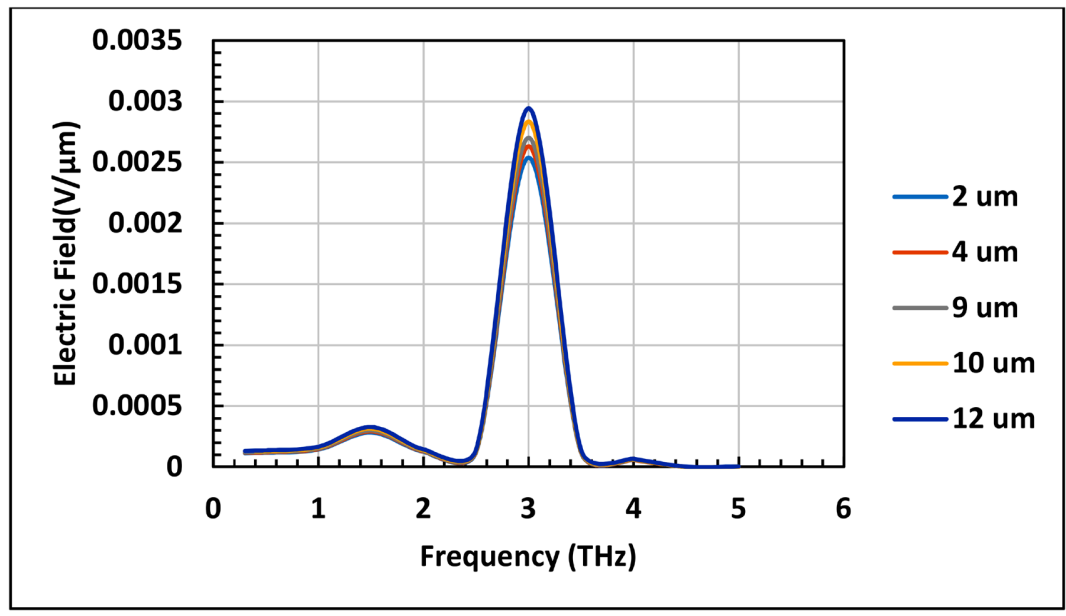

Figure 9. Electric field resulted from $\mathrm{Au} / \mathrm{SiC}$ antenna for different widths.

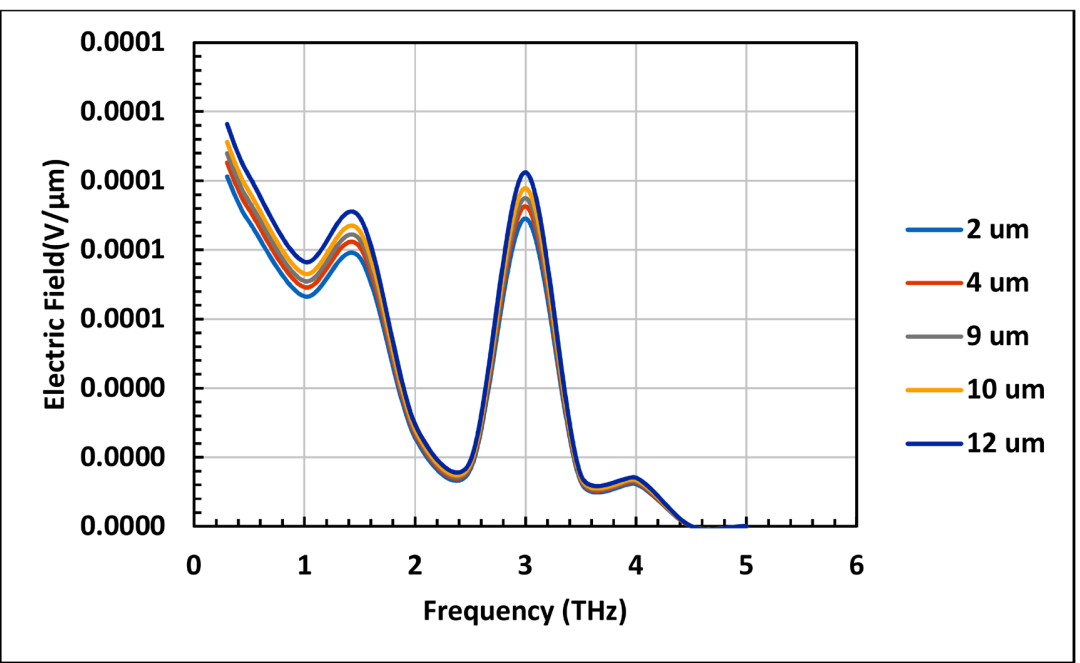

Figure 10. Electric field resulted from ITO/SiC antenna for different widths. 
the increase of charge accumulation across the gap and, as a result, to increasing the electric field. The second is the increase of the cross section normal to the surface, which increases the conductance, decreases the field loss, and consequently increases charge accumulation and the output field. However, it should be understood that the width does not fundamentally affect the output field, if the applied electric field is along the length.

For $\mathrm{Au} / \mathrm{SiC}$ antenna, at $3 \mathrm{THz}$, the field is $2.9 \mathrm{mV} / \mu \mathrm{m}$ for a $12 \mu \mathrm{m}$ width, and it is reduced to $2.6 \mathrm{mV} / \mu \mathrm{m}$ for a width of $2 \mu \mathrm{m}$, Figure 9 . For ITO/SiC antenna, at $3 \mathrm{THz}$, the field is $0.1 \mathrm{mV} / \mu \mathrm{m}$ for $12 \mu \mathrm{m}$ and it is reduced to $0.09 \mathrm{mV} / \mu \mathrm{m}$ for a width of $2 \mu \mathrm{m}$, Figure 10. Again the low field for ITO antenna is, partly, due to the low conductivity of ITO.

Changing the length of the antenna results in a more dramatic effect on the output electric field. The change of the antenna length changes the resonant frequency. This is expected, according to Equation (4). Figure 11 shows the effect of the length on the output of the antenna. For $L=8 \mu \mathrm{m}$, Equation (4) predicts a resonant frequency of $5.929 \mathrm{THz}$, which is beyond the range of the simulation (see the figure). For $L=12 \mu \mathrm{m}$, the equation predicts $f_{0}=3.95 \mathrm{THz}$, and this is in agreement with the simulated of $4 \mathrm{THz}$, as shown in the figure. For $L=$ $16 \mu \mathrm{m}$, the calculated value is $2.659 \mathrm{THz}$, which is close to the simulation value of about $3 \mathrm{THz}$. Similarly, for $L=20 \mu \mathrm{m}$, the calculation from Equation 4 gives a $f_{0}=2.37 \mathrm{THz}$ compared with $2 \mathrm{THz}$ from simulation. The difference increases for $L=25 \mu \mathrm{m}$, where the peak is at $1.9 \mathrm{THz}$ for calculation compared with about $1 \mathrm{THz}$ from simulation. This wide deviation can be attributed to the fact that the equation assumes the antenna metal is an ideal conductor with perfect structure reproducibility, which is not the case for practical antenna.

The same behavior of ITO antennas is shown in Figure 12. The figure shows that the magnitude at $f_{0}$ is much lower than that for $\mathrm{Au}$ antennas. The figure shows also that, for $L=16 \mu \mathrm{m}, f_{0}$ is also located at $3 \mathrm{THz}$. However, the secondary resonant frequency has double peaks with magnitudes similar to those at $f_{0}$. The double peaks may be due to overlapping between two adjacent peaks. For the Au antenna, the field is very low at $f_{0} / 2$ compared with the peak at $f_{0}$.

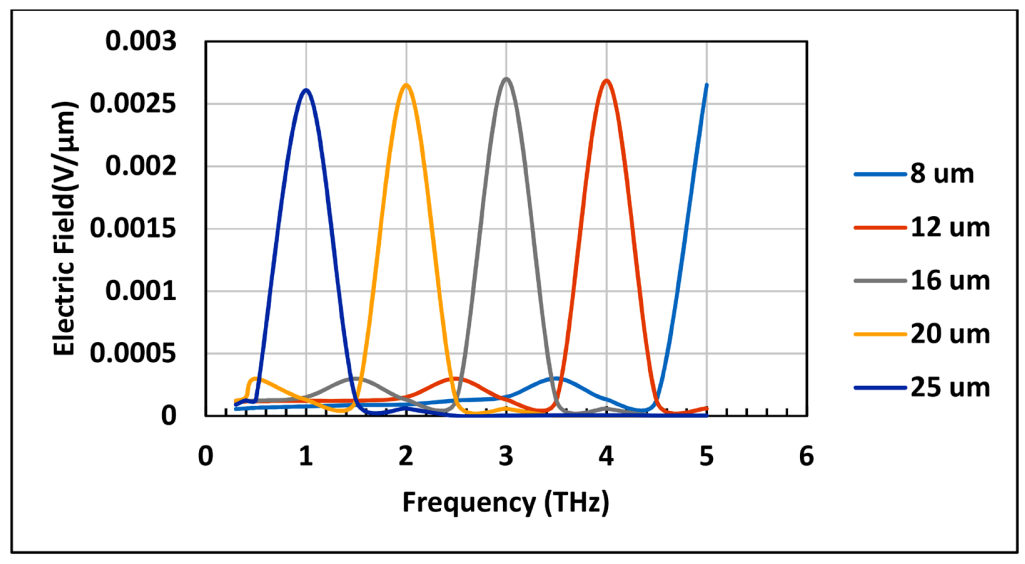

Figure 11. Electric field resulted from $\mathrm{Au} / \mathrm{SiC}$ antenna for different lengths. 


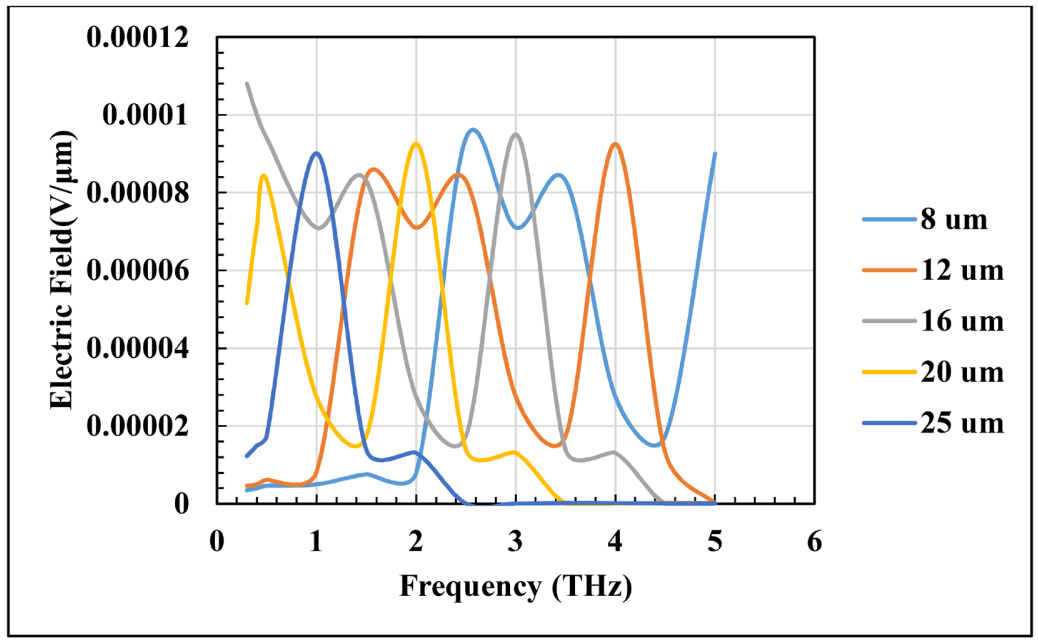

Figure 12. Electric field resulted from ITO/SiC antenna for different lengths.

Figure 11 and Figure 12 show that the peak magnitudes are not affected by the length. This behavior was not expected, since the increase in the length should increase the antenna area and, consequently, the radiation absorption. Increasing radiation absorption increases the output field. However, length has also an adverse effect on the output. The increase of the length results in increasing the distance the electrons must travel to reach the gap, and this decreases the output. Therefore, the two effects counter-balance each other, resulting in constant output with length.

For practical applications, antenna arrays can be built. Narrow band detection can be achieved using an array with a single antenna length. On the other hand, wide band detection can be achieved using an array with different lengths that results in resonant frequencies that cover the required band. In both cases, the antenna width, metal thickness, and gap separation are selected to optimize the antennas outputs. The topology of parallel and series connections should be designed to optimize the array output signal.

\section{Conclusion}

Bow-tie $\mathrm{THz}$ sensors and antennas can be built using Au or ITO on SiC substrates. Au can provide high conductivity and ITO can allow for vertical integration with optical devices. SiC has many advantages that makes it one of the best candidates for use in space and defense applications. This is because of its radiation hardness, temperature resistance, high thermal conductivity, and ability to integrate with electronic and optoelectronic systems. The study concludes that the output field decreases with the increase of the gap separation, but it increases with the increase of the antenna thickness. The study also shows that, although the antenna width does not fundamentally affect the output field, the increases of radiation absorption and metal conductance with the width indirectly increase the output field. On the other hand, the increase of the antenna length does not affect the output field, but it affects the resonant frequency. From this study, it is 
clear that an array for either narrowband detection or wideband detection can be built using these bow-tie antennas for space, defense, or other applications.

\section{Conflicts of Interest}

The authors declare no conflicts of interest regarding the publication of this paper.

\section{References}

[1] Wu, K., Cheng, Y.J., Djerafi, T. and Hong, W. (2012) Substrate-Integrated Millimeter-Wave and Terahertz Antenna Technology. Proceedings of the IEEE, 100, 2219-2232. https://doi.org/10.1109/JPROC.2012.2190252

[2] Sabaawi, M.A.A., Tsimenidis, C.C. and Sherif, B.S. (2012) Bow-Tie Nano-Array Antenna: Design and Optimization. 6th European Conference on Antennas and Propagation (EUCAP), 1975-1978. https://doi.org/10.1109/EuCAP.2012.6206439

[3] Sabaawi, A.M.A., Tsimenidis, C.C. and Sherif, B.S. (2013) Planar Bowtie Nanoarray for $\mathrm{THz}$ Energy Detection. IEEE Transactions on Terahertz Science and Technology, 3, 524-530. https://doi.org/10.1109/TTHZ.2013.2271833

[4] https://www.toptica.com/applications/terahertz-sensing/

[5] Wang, B.-X., He, Y.H., Lou, P.C. and Xing, W.H. (2020) Design of a Dual-Band Terahertz Metamaterial Absorber Using Two Identical Square Patches for Sensing Application. Nanoscale Advances, 2, 763-769. https://doi.org/10.1039/C9NA00770A

[6] Xu, W.D., Kono, J., Ping, J.F., Xie, L.J. and Ying, Y.B. (2020) Metamaterial-Free Flexible Graphene-Enabled Terahertz Sensors for Pesticide Detection at Bio-Interface. ACS Applied Materials \& Interfaces, 12, 44281-44287. https://doi.org/10.1021/acsami.0c11461

[7] Yu, Z., Perera, I.R., Daeneke, T., Makuta, S., Tachibana, Y., Jasieniak, J.J., Mishra, A., Bäuerle, P., Spiccia, L. and Bach1, U. (2016) Indium Tin Oxide as a Semiconductor Material in Efficient P-Type Dye-Sensitized Solar Cells. NPG Asia Materials, 8, e305. https://doi.org/10.1038/am.2016.89

[8] Properties of Silicon Carbide. http://www.ioffe.ru/SVA/NSM/Semicond/SiC/thermal.html.

[9] Wu, Y.M., Le-Wei, L. and Liu, B. (2010) Geometric Effects in Designing Bow-Tie Nanoantenna for Optical Resonance Investigation. The Asia-Pacific International Symposium on Electromagnetic Compatibility, Beijing, April 2010, 12-16.

[10] Wu, Y.M., Le-Wei, L. and Liu, B. (2010) Gold Bow-Tie Shaped Aperture Nanoantenna: Wide Band Near-Field Resonance and Far-Field Radiation. IEEE Transactions on Magnetics, 46, 1918-1921. https://doi.org/10.1109/TMAG.2010.2043063

[11] Ordal, M.A., Long, L.L., Bell, R.J., Bell, S.E., Alexander, R.W. and Ward, C.A. (1983) Optical Properties of the Metals Al, Co, Cu, Au, Fe, Pb, Ni, Pd, Pt, Ag, W, and Ti in the Infrared and Far Infrared. Applied Optics, 22, 1099-1199. https://doi.org/10.1364/AO.22.001099

[12] Gonzalez, F.J., Alda, J., Simon, J., Ginn, J. and Boreman, G. (2009) The Effect of Metal Dispersion on the Resonance of Antennas at Infrared Frequencies. Infrared Physics \& Technology, 52, 48-51. https://doi.org/10.1016/j.infrared.2008.12.002

[13] Sindooja, B.V. and Mary, T.A.J. (2014) Design of Modified Bowtie Antenna for Wireless Applications. International Journal of Engineering Research \& Technology (IJERT), 3, 783-786. 